A

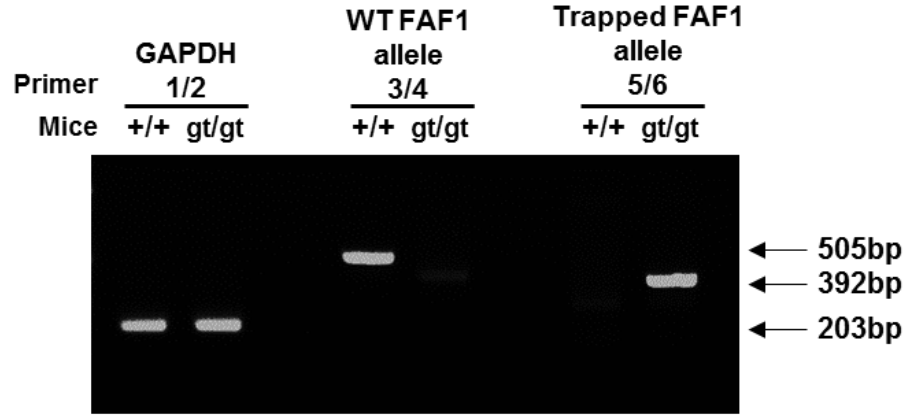

C

Mouse GAPDH

1: 5' TGACCACAGTCCATGCCATC 3'

2: 5' GACGGACACATTGGGGGTAG 3

\section{WT FAF1 allele}

3: 5' TCCATCATCAGCACCAGATA 3'

4: 5' GCATGTGTGAGATGGTTCAA 3'

Trapped FAF1 allele

5: 5' GACGTCTCGTTGCTGCATAA 3'

6: 5' CAGCAGCAGACCATTTTCAA 3'

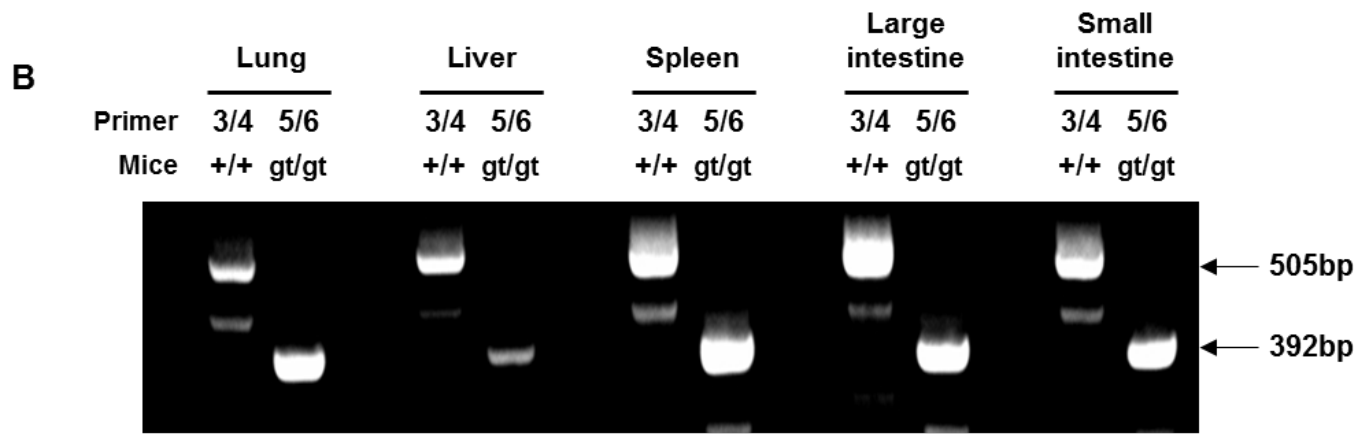

$\mathrm{FAF}^{+/+}$

FAF $1{ }^{\mathrm{gt}} / \mathrm{gt}$
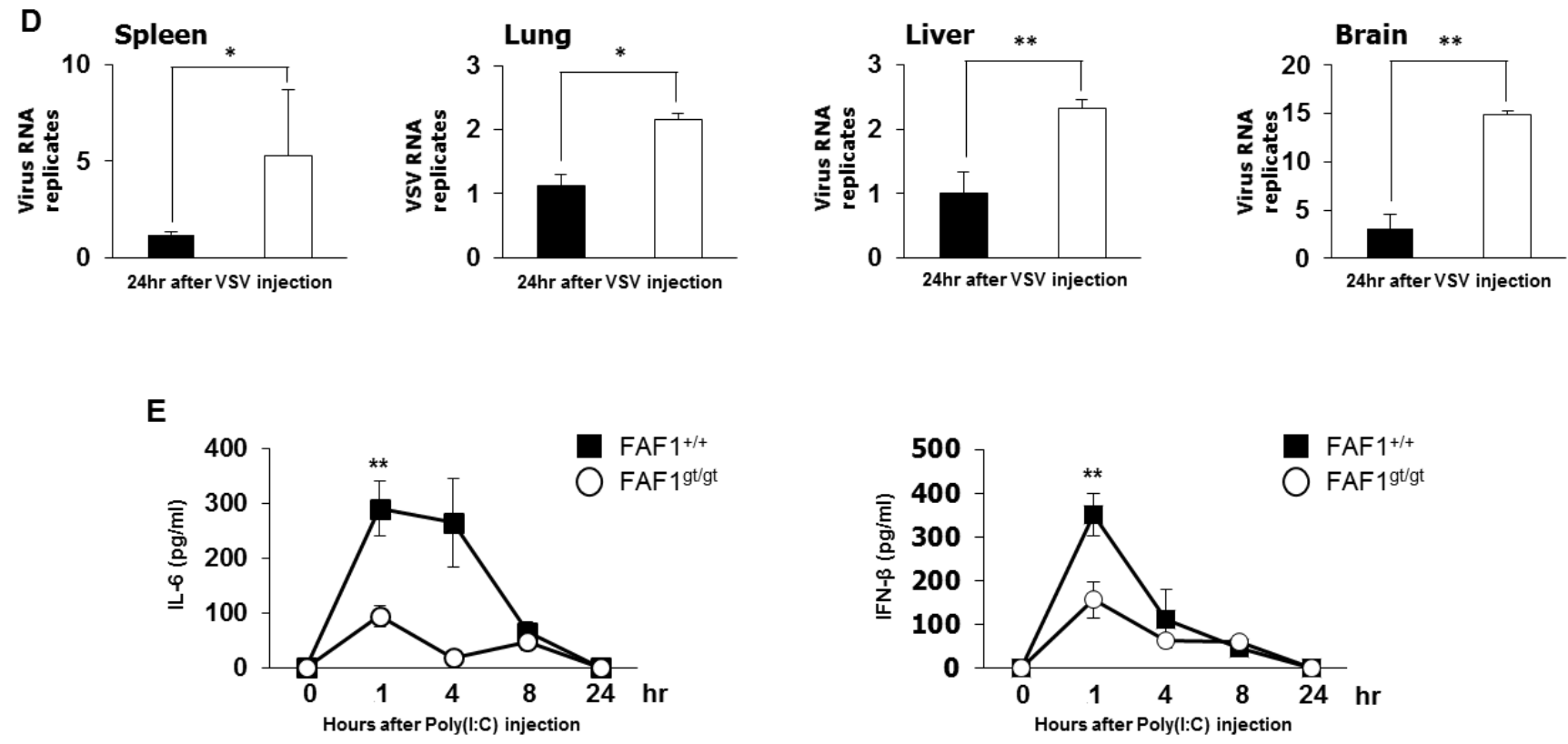

S1 Fig. Immune responses were decreased in FAF1 ${ }^{\text {gtgt }}{ }^{\text {mice }}$ upon VSV-Indiana infection and Poly (I:C) treatment. (A and C) The genotypes of the wild-type $\left(\mathrm{FAF}^{+/+}\right)$and $\mathrm{FAF} 1$-knockdown $\left(\mathrm{FAF} 1^{\mathrm{gtg} t}\right)$ mice were conducted by generating PCR fragments from tail DNA (A) and from isolated organs (lung, liver, spleen, large intestine and small intestine) (B). PCR fragments were generated with the primers (C) of WT FAF1 allele from FAF1 ${ }^{+/+}$mice and trapped FAF1 allele from FAF1 ${ }^{\mathrm{gtgt}}$ mice. Mouse GAPDH primers were used as an internal reference gene (positive control). (D) FAF1 ${ }^{+/+}(n=4)$ and FAF1 ${ }^{\text {gtgt }}(n=4)$ mice whole organs (spleen, lung, liver and brain) were collected at 24 hpi of VSV-Indiana $\left(2 \times 10^{8} \mathrm{pfu} /\right.$ mouse) via tail-vein injection. The viral load in supernatants of homogenized organs were measured by qRT-PCR. Data represent mean \pm SD. ${ }^{*} P<0.05$ and ${ }^{* *} P<0.01$ as compared between the indicated groups (Student's t test). (E) FAF1 ${ }^{+/+}(\mathrm{n}=5)$ and FAF1 ${ }^{\mathrm{gtgt}}(\mathrm{n}=5)$ mice were injected with Poly (I:C) (200 $\mu \mathrm{g}$ per mouse) via tail-vein injection. Sera were collected from the mice at indicated time points and IL-6 and IFN- $\beta$ were measured by ELISA. Data represent mean \pm SD. ${ }^{* *} P<0.01$ as compared between the indicated groups (Student's $\mathrm{t}$ test). 